\title{
Decisions to Enter and Leave Skilled Nursing Facilities for Post-Acute Rehabilitation: \\ A Qualitative Study
}

Daniel Chimitt ${ }^{1}$, Jennifer Carnahan ${ }^{2,3}$

Indiana University School of Medicine ${ }^{1}$, Regenstrief Institute ${ }^{2}$, Indiana University Center for Aging Research ${ }^{3}$

\section{Background and Hypothesis:}

Approximately $40 \%$ of patients aged $80+$ enter a Skilled Nursing Facility (SNF) following a hospitalization. SNFs can be used as "safety nets" to expedite the discharge process of older adults and it can be difficult to pinpoint how and who made the decision for a hospitalized older adult to discharge to a SNF.

This project examines the factors that drive older adults to enter and leave a SNF for their rehabilitation care.

\section{Project Methods:}

Interview transcripts from a qualitative study with patients and their caregivers were used to examine factors influencing admission to and discharge from SNFs. Baseline interviews were conducted within two to seven days after returning home from a SNF stay followed by a follow up phone call one to two weeks after the initial interview. Transcripts and audio files were coded (using NVivo version 12+) for major themes. Interviews were analyzed using a constant comparative method to elicit themes of interest to interviewees.

\section{Results:}

There were 24 baseline interviews and $X$ follow up interviews performed with a total of 24 patients and 15 caregivers. The primary theme identified was that patients perceived a loss of autonomy when considering the decision-making process. $75 \%(18 / 24)$ patients or their caregivers felt the healthcare team told them they must go to a SNF for their rehabilitation. 38\% $(9 / 24)$ patients or caregivers felt they had no choice but to leave due to insurance coverage and $50 \%(12 / 24)$ stated that they needed more time.

\section{Potential Impact:}

To achieve better patient outcomes, one must understand both the purpose of skilled nursing facilities and also how patients and their families are feeling as they transition through this uncertain period of their lives. Restoring a patient's sense of autonomy will foster better patienthealthcare relationships and improve trust in the system. 